\title{
Stress in professional care-givers working with patients with dementia: a hypothesis-generating study
}

\author{
Gianluca Isaia1, Marco Astengo¹, Giovanni Carlo Isaia1, Mario Bo1, Giorgetta Cappa1, Simona \\ Mondino1$^{1}$, Giulia Nobili1 ${ }^{1}$, Valerio Dimonte ${ }^{2}$, Lucie Ernestine Nkouka1 and Massimiliano Massaia1 \\ 1Department of Medical and Surgical Disciplines, Geriatric Section, University of Torino, San Giovanni \\ Battista Hospital, Torino, 2Public Health and Microbiology, University of Torino, Torino, Italy
}

\begin{abstract}
Background and aims: Caregiving can be extremely stressful, especially when patients' ability to communicate is impaired. While the stress undergone by relatives assisting their loved ones has been widely investigated, fewer data can be found about the stress in healthcare professionals. The aim of this study is to evaluate whether a specific training course could be related to a reduction in the levels of stress of professional care-givers working with patients suffering from dementia. Methods: Work-related levels of stress of study participants were evaluated with the Staff Stress Measure Dementia Care Scale, at baseline and four months after completion of an eight-month training course. Results: We found no significant correlation between care-givers' age, gender, marital status, years of employment or perceived economic status, and their stress levels at baseline. $\mathrm{Pa}$ tients' characteristics were not related with care-givers' stress at baseline. The mean level of stress was significantly reduced $(34.64 \pm 4.15$ vs $26.64 \pm 3.82, \mathrm{p}<0.001)$ between baseline and the study endpoint. Conclusions: Increased knowledge of management of patients affected by dementia could help professional care-givers to reduce their work-related stress. Our results add to the evidence of the benefit of personnel support in reducing levels of stress at work.

(Aging Clin Exp Res 2011; 23: 463-469)

${ }^{\circ}$ 2011, Editrice Kurtis
\end{abstract}

\section{INTRODUCTION}

In developed countries, the number of older people needing healthcare and long-term care is increasing. Functionally and cognitively impaired older people put a heavy burden on healthcare workers. Nursing staff in residential-aged care settings have a difficult and challenging job (1). People employed in nursing homes and long-term facilities work long hours, are poorly paid, and receive minimal benefits, while the patients they care for present an elevated burden of care. Caregiving can be extremely stressful, especially when the patients' ability to communicate is impaired (2). Novak and Chappell observed that, the worse the cognitive impairment of the residents, the more severe the level of stress of the healthcare professionals caring for them (3). At the same time, several studies have reported that heavier work loads have led to increased time pressure among nursing staff, resulting in higher levels of stress $(4,5)$.

Healthcare professionals have been found to respond to their inadequacy not only with tension, anxiety and avoidance, but also with decreased self-esteem and job satisfaction. This can lead to depression or burn-out, determining what is usually known as "work-related stress".

Stress has been defined as a subjective psycho-physiological state characterized by a combination of high arousal and displeasure (6). It is considered to result from an imbalance between the demands of the workplace and an individual's ability to cope with them.

Stress often results in adverse physiological and psychological outcomes for both care-givers and recipients (7, 8 ) and high job strain is associated with increased risk of a multitude of illnesses among workers $(9,10)$.

While the stress of relatives assisting their loved ones has been widely investigated (11-13), fewer data can be found about stress in healthcare professionals, with the exception of personnel working with cancer patients (14).

During the last few decades, some educational programs, aiming to reduce the stress of professional caregivers, have been developed. Although the reduction in

Key words: Dementia Care, nursing education, nursing homes, nursing practice, stress.

Correspondence: Gianluca Isaia, MD, Department of Medical and Surgical Disciplines - Geriatric Section, University of Torino, San Giovanni Battista Hospital, Corso Bramante 88, I-10126 Torino, Italy.

E-mail: gianlucaisaia@yahoo.it

Received July 20, 2010; accepted in revised form November 30, 2010.

First published ahead of print December 15, 2010 as DOI: 10.3275/7412 
shift length and the number of patients each healthcare worker must take care for would probably be effective in ameliorating the quality of working life, these goals are difficult to achieve in healthcare systems with limited economic resources. Instead, training courses, based on cognitive, emotional and behavioral approaches are more feasible and could help to reduce professional care-givers' stress by providing them with better knowledge and more realistic expectations.

Our main aims were to identify which items of a specific staff stress scale are mainly compromised in professional care-givers and which items are mainly modified after a specific training course, aimed at improving knowledge in the management of patients affected by dementia. The study also aimed at highlighting the problem of the burden of professional care-givers in long-term facilities by reporting the main features of patients they have to care for and by searching for possible relationships between stress and patients' characteristics.

\section{METHODS}

The study was performed in two nursing homes in Turin, northern Italy, belonging to the Department of Geriatrics of the city's University Hospital and hosting 200 non-independent older subjects. All registered nurses and nurse assistants working in the two facilities were asked to participate in the present study.

Data on gender, age, marital status, years of employment and perceived economic status of the professional care-givers were collected. Data on gender, age, marital status, years of employment, perceived economic status and levels of stress were also collected for non-participating nurses.

All participants underwent baseline evaluation of their level of stress with the Staff Stress Measure Dementia Care (15), a scale specifically designed and validated for healthcare professionals working with patients affected by Alzheimer's disease or similar degenerative mental conditions. This scale is composed of 20 items; each item has five possible answers: $0=$ never, $1=$ a little, $2=$ sometimes, $3=$ often, and $4=$ always. The score is calculated by the sum of these items, higher scores indicating higher levels of work-related stress.

All study participants underwent a training course designed to increase their knowledge about the care of older, cognitively and functionally impaired subjects and to enhance their capability to cope with stress-generating situations. The course lasted eight months, from January to August 2006, and consisted of two hours of training per week, for a total of 32 hours of theoretical lessons and 32 hours of role-playing exercises and exchange of experiences. The main topics of the theoretical lessons were as follows: aging and cognitive impairment, symptoms and stages of dementia, management of behavioral and psychological symptoms related to cognitive impairment, nutritional issues in patients affected by dementia, pain evaluation and control in patients suffering from dementia, strategies for successful communication with patients with dementia, impact of dementia on the quality of life of the care-giver, psychosocial and ethical issues associated with dementia, and the continuity of care. In each lesson, the specific topic was developed with attention to the stress-related aspects and possible interventions useful in reducing working stress. Theoretical lessons were completed with presentation and discussion of clinical cases and video clips. At the end of each lesson, participants were given a paper summarizing the issues discussed and some review questions. A total of 16 role-playing exercises were scheduled: participants were divided into five groups of ten people each. During each practical lesson, each group role-played one scenario. Each scenario was presented by two participants with the help of the teacher, and with an actor playing the role of the patient. After the first 10 scenarios, the actor was substituted with a patient affected by dementia. Scenarios aimed to put the topics presented during theoretical lessons into practice.

The training course was held by a senior physician working at the memory clinic of the Department of Geriatrics of a university teaching hospital (San Giovanni Battista, Turin, Italy), by a nurse with expertise in the care of patients suffering from dementia, and by a psychologist skilled in group training and role-playing. Two theoretical lessons (nutritional issues and pain evaluation and control) were given by the nutrition and pain specialists, respectively. Table 1 lists the essential characteristics of the course.

At the end of each lesson, each participant could ask their teachers questions on the specific lesson they had attended or other topics of the past lessons or during their daily work. Moreover, the nurse and the physician holding the training course devoted one hour per week to the students, between the end of the course and the follow-up evaluation, in order to answer any questions and give work support in general.

One year after baseline evaluation, professional caregivers underwent the same evaluation of stress that they did at baseline. Each item of the Staff Stress Measure Dementia Care measured at the end of the study was compared with the same item measured at baseline.

Data on patients' characteristics (age, gender, grade of cognitive impairment, functional status, entity and frequency of behavioral disturbances) were also collected.

The Clinical Dementia Rating Scale (CDR) was used to evaluate the degree of cognitive impairment. This is a fivepoint scale, with CDR-0 indicating no cognitive impairment, and the remaining four scores indicating progressively more severe stages of dementia $(C D R-0=a b s e n t$, CDR- $0.5=$ very mild, CDR- $1=$ mild, CDR- $2=$ moderate, CDR-3=severe) $(16,17)$. The Activity of Daily Living 
Table 1 - Schedule of course.

\begin{tabular}{|c|c|c|c|}
\hline & & Duration & \multirow{9}{*}{$\begin{array}{l}16 \text { role-playing exercises } \\
2 \text { hours each } \\
10 \text { participants per group }\end{array}$} \\
\hline Aging and cognitive impairment & \multirow{8}{*}{ 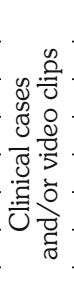 } & 4 hours & \\
\hline Symptoms and stages of dementia & & 4 hours & \\
\hline Management of behavioral and psychological symptoms related to cognitive impairment & & 4 hours & \\
\hline Nutritional issues in patients affected by dementia & & 4 hours & \\
\hline Pain evaluation and control in patients suffering from dementia & & 4 hours & \\
\hline Strategies for successful communication with patients with dementia & & 4 hours & \\
\hline Impact of dementia on quality of life of caregiver & & 4 hours & \\
\hline Psychosocial and ethical issues associated with dementia and continuity of care & & 4 hours & \\
\hline
\end{tabular}

Scale (ADL) was used to evaluate the functional status of patients. The Cohen-Mansfield Agitation Inventory (CMAI) was used to estimate behavioral disturbances. This 29-item questionnaire is specific for patients with dementia, and must be completed by a formal care-giver (18), and defines agitation on a 7 -point frequency scale $\left(0=\right.$ never, $6=$ sev- $^{-}$ eral times per hour).

All data were collected by a geriatrician with more than 5 years of experience in geriatric research, who was not aware of the aim of the study and who had no role either in study analyses or in the care of patients. Informed consent for study participation was obtained from each professional care-giver and from each patient or proxy (closest relative or legal guardian). The study was approved by the Hospital Ethical Committee.

\section{Statistical analysis}

Statistical analysis was performed with the SPSS 15.0 package for Windows. A descriptive analysis of continuous and categorical variables was performed. The Independent-Samples $t$-test procedure was used to evaluate differences in mean values between groups. The Paired-Samples $t$-test procedure was used to evaluate differences between baseline and follow-up; $p<0.05$ indicates statistical significance. The effect size between pre- and posttraining Staff Stress Measure Dementia Care scale scores was measured with Cohen's d (95\% confidence interval). ANOVA was also used to evaluate differences in Staff Stress Measure Dementia Care scale scores between baseline and follow up.

Data are presented as means \pm SD $(95 \%$ confidence intervals) or as percentages in the corresponding categories.

\section{RESULTS}

Fifty professional care-givers participated in the present study. Twenty-seven of the 40 professional care-givers were employed in the first nursing home and 23 of the 40 professional care-givers employed in the second one accepted participating in the study. Of the 30 healthcare workers who did not participate, 28 were not interested in the study and two dropped out before it ended. The mean age of the care-givers enrolled was $42.9 \pm 8.7$ years (range 25-63), 42 (84\%) were women, 32 (64\%) were married, $10(20 \%)$ unmarried, $1(2 \%)$ was a widow, and $7(14 \%)$ were divorced. They had worked in the facility for $13.6 \pm 10.0$ years (range 3-35 yrs). Salaries were considered insufficient by $62 \%$ of subjects in the study. The Staff Stress Measure Dementia Care Scale scores, indicating professional care-givers' levels of stress, were 34.6 \pm 4.1 and 26.6 \pm 3.8 at baseline and after one year of follow-up, respectively ( $t=10.03, p<0.001$; Cohen's d effect size $=1.93,95 \%$ confidence interval 1.44-2.39). Table 2 shows mean values of the single items in the Staff Stress Measure Dementia Care Scale at baseline and after one year of follow-up.

Thirty (37.5\%) of the 80 nurses working in the two nursing homes did not wish to participate in the study. This may reflect the difficulty of obtaining good adherence to educational courses in real-life settings. However, no significant differences were observed between participating and non-participating nurses as regards age $(\mathrm{t}=1.67$, $p=\mathrm{ns}$ ), gender (Chi-Square $=3.30, p=\mathrm{ns}$ ), marital status (Chi-Square $=2.09, p=n s)$, years of employment $(t=2.89$, $p=n s$ ), perceived economic status (Chi-Square $=2.77$, $p=n s)$ and level of work-related stress ( $t=0.97, p=n s)$.

The patients cared for in the two nursing homes at the beginning of the study were 212; their mean age was $81.7 \pm 8.7$ years (range 62-99 yrs); $132(62 \%)$ were women; mean CDR 2.5 \pm 0.5 (97\% classified as CDR-2 or CDR-3); mean ADL 5 \pm 1.2 ; mean CMAI 21.9 \pm 19.2 .

We did not find significant correlations between caregivers' levels of stress (Staff Stress Measure Dementia Care score) and their age ( $\mathrm{r}=-0.28, p=\mathrm{ns})$, gender (ChiSquare $=2.08, p=n s$ ), marital status (Chi-Square $=4.02$, $p=\mathrm{ns}$ ) or perceived payment (Chi-Square $=3.18, p=\mathrm{ns})$, at baseline.

Neither the functional nor cognitive status of patients were related to care-givers' levels of stress at baseline $(\mathrm{r}=-0.17, p=\mathrm{ns}$ and $\mathrm{r}=-0.26, p=\mathrm{ns}$ respectively). Moreover, behavioral disturbances were not statistically related to care-giver stress $(\mathrm{r}=0.61, p=0.672)$. 
Table 2 - Mean values of items in Staff Stress Measure Dementia Care Scale at baseline and at one year of follow-up.

\begin{tabular}{|c|c|c|c|c|}
\hline \multicolumn{2}{|c|}{ Items } & \multirow{2}{*}{$\begin{array}{c}\begin{array}{c}\text { Baseline } \\
\text { mean } \pm \text { SD }\end{array} \\
1.26 \pm 1.27\end{array}$} & \multirow{2}{*}{$\begin{array}{c}\text { One year follow-up } \\
\text { mean } \pm \text { SD }\end{array}$} & \multirow{2}{*}{$\begin{array}{l}\text { p-value } \\
\mathrm{ns}\end{array}$} \\
\hline 1. & Their forgetfulness really gets on my nerves & & & \\
\hline 2. & I believe these residents should do more for themselves & $1.90 \pm 1.42$ & $1.26 \pm 0.80$ & 0.007 \\
\hline 3. & I am afraid these residents will get violent and hurt someone & $1.34 \pm 1.56$ & $1.42 \pm 1.18$ & ns \\
\hline 4. & I get tired of repeating things to them so often & $2.50 \pm 1.42$ & $1.36 \pm 1.17$ & $<0.001$ \\
\hline 5. & Their families just do not appreciate what we do for these people & $1.68 \pm 1.62$ & $1.9 \pm 1.18$ & ns \\
\hline 6. & Their babbling and rambling speech gets on my nerves & $1.96 \pm 1.37$ & $1 \pm 0.95$ & $<0.001$ \\
\hline 7. & It is very hard for me to communicate with these people & $2.30 \pm 1.20$ & $1.2 \pm 0.97$ & $<0.001$ \\
\hline 8. & I get angry when they deny problems and blame others for things & $2.34 \pm 1.62$ & $0.94 \pm 0.97$ & $<0.001$ \\
\hline 9. & I really get tired working with these residents & $2.56 \pm 1.18$ & $1.62 \pm 1.10$ & $<0.001$ \\
\hline 10. & It is hard to accept what is happening to these residents & $1.74 \pm 1.26$ & $1.44 \pm 0.99$ & ns \\
\hline 11. & I get frustrated and angry working with these people & $2.24 \pm 1.42$ & $1.25 \pm 0.93$ & $<0.001$ \\
\hline 12. & I think more medication would make it easier to help them & $1.16 \pm 1.33$ & $1.28 \pm 1.21$ & ns \\
\hline 13. & I have trouble talking to their families & $0.90 \pm 1.31$ & $1.18 \pm 1.08$ & ns \\
\hline 14. & I take my work with them home with me & $0.80 \pm 1.16$ & $0.76 \pm 0.98$ & ns \\
\hline 15. & I feel these residents should appreciate our help more & $2.20 \pm 1.56$ & $0.82 \pm 0.90$ & $<0.001$ \\
\hline 16. & It worries me these people will wander off & $0.96 \pm 1.26$ & $1.94 \pm 1.30$ & $<0.001$ \\
\hline 17. & I feel it takes too long to do things for them & $1.90 \pm 1.37$ & $1.66 \pm 1.15$ & 0.06 \\
\hline 18. & It bothers me how helpless these residents become & $2.14 \pm 1.47$ & $1.46 \pm 1.05$ & 0.005 \\
\hline 19. & I believe we should have more training to work with them & $1.42 \pm 1.44$ & $1.66 \pm 1.22$ & ns \\
\hline 20. & It is difficult to explain their behavior to other residents or families & $1.32 \pm 1.56$ & $1.4 \pm 1.07$ & ns \\
\hline
\end{tabular}

SD: standard deviation; ns: non-significant.

Although no correlations between work-related levels of stress and care-givers' characteristics were observed at baseline, the effect of possible confounders was evaluated by ANOVA: the decrease in levels of work-related stress at one year remained statistically significant after forcing age, gender, marital status, years of employment and perceived economic status in the model $(\mathrm{F}=12.67$, $p<0.001$ ).

The items of the Staff Stress Measure Dementia Care Scale that had shown the highest scores at baseline also showed the most consistent improvements after one year of follow-up [items number 9 (I really get tired working with these residents), number 4 (I get tired of repeating things to them so often), number 8 (I get angry when they deny problems and blame others for things), number 7 (It is very hard for me to communicate with these people) and number 11 (I get frustrated and angry working with these people)]. Only item number 16 (It worries me these people will wander off) showed a higher score after one year than at baseline.

After one year of follow-up, the items with the highest scores were items 16, 5 (their families just do not appreciate what we do for these people), 17 (I feel it takes too long to do things for them) and 19 (I believe we should have more training to work with them). Conversely, items with the lowest scores were numbers 8 (I get angry when they deny problems and blame others for things), 15 (I feel these residents should appreciate our help more) and 14 (I take my work with them home with me).

\section{DISCUSSION}

The present study evaluated the levels of work-relatedstress of professional care-givers working in two nursing homes before and after having participated in a training course targeted to the management of older, cognitively impaired patients.

Working with patients affected by dementia has been shown to be very stressful $(2,3)$ : affected subjects can be aggressive or listless and have difficulty in creating relationships, caring may evoke feelings of meaninglessness (19) and, in particular situations, such as the case of disruptive behavior, care-givers may feel ineffectual (20).

Our results cannot relate stress to any one of the outcomes described. The levels of stress of professional care-givers do not correlate with patients' degree of cognitive or functional impairment, or their frequency of behavioral disturbances. In addition, age, gender, marital status and perceived economic status of care-givers do not influence their levels of stress.

One explanation is that we have not accounted for other variables which are involved in stress generation, and this may be a limitation of the study, although the most important variables were collected. Another explanation is that staff stress is mainly caused by the individual perception that professional care-givers have on their patients. 
A critical interpretation of our results suggests that levels of stress at baseline are related to a lack of understanding of the typical features and needs of patients affected by dementia: after the training course, not only was global stress reduced, but the reasonable fear of patients wandering off (item 16) took the place of the frustration deriving from communication difficulties (items 4,7 and 8 ) adding to nurses' worries. It is also important to emphasize that the CMAI scale did not give high results, describing a population without important behavioral problems. Performing a similar study with a higher number of patients may produce different results.

It should be emphasized that the course was not specifically structured to achieve better performance on the stress test. The fact that professional care-giver stress was not related to several variables evaluated allows us to hypothesize the role of individual perceptions and knowledge. However, the weak design of the present study and the low number of participants do not allow us to draw firm conclusions.

Some studies $(21,22)$ have described strategies for stress management, often focusing on specific illnesses. These interventions are usually subdivided into personnel support interventions, aiming at teaching personnel how best to deal with a variety of stressful situations, and environmental management interventions, aiming at reducing the sources of stress in the workplace. As reported by Mimura et al. (23), more evidence is available for the effectiveness of personnel support than of environmental management. Lack of support can be either a stressor or an effective coping strategy, depending on its presence or absence. The lack of managerial support to and direct leadership of staff induces stress. Supervision aimed at implementing personalized planned care has shown decreased incidence of burnout and increased job satisfaction among samples of nurses $(24,25)$. Conversely, other authors $(26,27)$ have reported that the lack of support at work did not contribute significantly to nurses' levels of stress.

The field of educational interventions for nurses and professional care-givers is expanding. However, programs based only on lectures are often seen as boring by their recipients. A review of the literature gives no indication why role playing would be effective in achieving the desired outcomes but, in our opinion, the opportunity of alternating theoretical lessons and role-playing may be more appealing and more effective, producing better learning results.

The results of the present hypothesis-generating study suggest that an educational course consisting of both theoretical lessons and practical training sessions is effective in reducing personnel's level of work-related stress, in a sample of nurses dealing with cognitively and functionally impaired older patients.

Our results are partially in contrast with the findings of other authors who identified excessive work loads, long duty hours, financial problems, conflict between professional and personal life, and feeling responsible for patients' outcomes as some of the main determinants of stress (28-30). These differences may be explained in different ways. First, previous studies were conducted on samples of health professionals working with cancer patients rather than with subjects suffering from dementia. We speculate that a training course is more likely to help professional care-givers to cope with issues in communication with patients with dementia, than with the lack of hope for terminal cancer patients. Second, other studies used other tools to measure work-related stress. We chose to use the Staff Stress Measure Dementia Care Scale, because it was specifically designed to measure stress in healthcare workers dealing with patients affected by Alzheimer's disease in nursing homes and day care centers.

One objection to the present study may be advanced by looking the relatively low Staff Stress Measure Dementia Care scale scores. A relatively low score (34.6 points) would not prompt a training course for nurses. However, many items report a mean score of over 2 points, meaning "frequently". In particular, some of these items, such as "I get tired of repeating things to them so often", "I get angry when they deny problems and blame others for things" and "I feel these residents should appreciate our help more" evidence a lack of knowledge of the management of patients with dementia. The same consideration may be made for the item "It worries me these people will wander off", with low scores at baseline and higher ones after the course. The hypothesized lack of knowledge of management of these patients was the starting-point of the course development. The course was in fact structured to give a better knowledge of management and the most important features of patients affected by dementia. The aim of the course was not to reduce care-givers' stress directly, but to improve their knowledge in order to achieve a reduction in stress. We did not mention self-controlling strategies, escape avoidance strategies or social support, as reported in other studies (31). The lack of these topics may be considered a limitation in a study aimed at reducing stress, but not in a hypothesis-generating study aimed at improving knowledge and, secondarily, reducing stress. Despite the weak study design, our results suggest that lack of awareness may be involved in work-related stress.

In support of the hypothesis of our study, several manuscripts have been published on the role of improving knowledge by practice $(32,33)$. The literature shows that an experiential approach is an effective strategy for teaching students and nurses (34). The uniqueness of our intervention may have been the combination of experiences, i.e., role-playing and lectures, and probably also the length of the intervention (8 months). It may be ar- 
gued that the possibility of having available special professional figures able to answer specific questions would be more efficacious than a shorter course.

Our study presents some important limitations. Because of the relatively small sample size, our study should be regarded to as hypothesis-generating. However, the complexity of the proposed intervention makes it hard to recruit very large samples of subjects. Another critical aspect was the level of nurses' compliance with the course. We did not perform specific evaluation of this, but caregivers were not obliged to follow the course and there was no advantage as regards their vocation. Moreover, each participant could withdraw from the course without any consequences. The fact that only two participants dropped out may reflect a high level of compliance, although it remains a limitation for the study.

Nor did we compare the effectiveness of one intervention with another one or with "usual care". However, the nurses had worked for a long time in the two nursing facilities and this fact makes it very unlikely that stress levels would decrease as a result of the personnel gaining experience. A follow-up of one year may be too short to evaluate whether the effects of the course are permanent or at least long-lasting. Lastly, the different educational systems for nurses in different countries may make generalization of our results difficult.

\section{CONCLUSIONS}

Our results add to the evidence of the benefit of personnel support in reducing levels of work-related stress. Nurses working with patients affected by dementia may not have specific training in this field. Sometimes they learn their work by doing it, but, in our opinion, some peculiarities of patients affected by dementia require specific attention and teaching. Care-givers' reactions against some little-known aspects of dementia may be higher than expected and could give rise to stressing emotions. This does not mean that nurses fail during their work, but it may be hypothesised that better knowledge could help professional care-givers in their work. This goal could also have positive effects on patients.

The limitations inherent in our study do not allow us to make definitive recommendations on a specific approach toward improving working conditions and reducing healthcare workers' stress. Further research, on larger samples of professional care-givers and comparing different interventions, is needed.

\section{REFERENCES}

1. Edvardsson D, Sandman PO, Nay R, Karlsson S. Predictors of job strain in residential dementia care nursing staff. J Nurs Manag 2009; 17: 59-65.

2. Bjorkhem K, Olsson A, Hallberg IR, Norberg A. Caregivers' experience of providing care for persons suffering from dementia living at home. Scand J Prim Health Care 1992; 10: 53-5.

3. Novak M, Chappell N. Nursing assistant burnout and the cogni- tively impaired elderly. Int J Aging Hum Dev 1994; 39: 105-12.

4. Denton M, Zeytinoglu IU, Davies S, Lian J. Job stress and job dissatisfaction of home care workers in the context of health care restructuring. Int J Health Serv 2002; 32: 327-57.

5. Morgan DG, Semchuk KM, Stewart NJ, D'Arcy C. Job strain among staff of rural nursing homes: a comparison of nurses, aides, and activity workers. J Nurs Admin 2002; 32: 152-61.

6. Ruotsalainen J, Serra C, Marine A, Verbeek J. Systematic review of interventions for reducing occupational stress in health care workers. Scand J Work Environ Health 2008; 34: 169-78.

7. Schulz R, Beach SR. Caregiving as a risk factor for mortality: the Caregiver Health Effects Study. JAMA 1999; 282: 2215-9.

8. Noelker LS. The backbone of the long-term care workforce. Generations 2001; 25: 85-91.

9. Kivimaki M, Leino-Arjas P, Luukkonen R, Riihimäki H, Vahtera J, Kirjonen J. Work stress and risk of cardiovascular mortality: Prospective cohort study of industrial employees. BMJ 2002; 325: 857-60.

10. Kuper H, Marmot M. Job strain, job demands, decision latitude, and risk of coronary heart disease within the Whitehall II study. J Epidemiol Community Health 2003; 57: 147-53.

11. Schulz R, Leino-Arjas $\mathrm{P}$, Luukkonen $\mathrm{R}$, Riihimäki $\mathrm{H}$ et al. Dementia patient suffering and caregiver depression. Alzheimer Dis Assoc Disord 2008; 22: 170-6.

12. Haley WE, Levine EG, Brown SL, Berry JW, Hughes GH. Psychological, social and health consequences of caring for a relative with senile dementia. J Am Geriatr Soc 1987; 35: 405-11.

13. Hall GR. Care of the patient with Alzheimer's disease living at home. Nurs Clin North Am 1988; 23: 31-46.

14. Bourbonniere M, Kagan SH. Nursing intervention and older adults who have cancer: specific science and evidence based practice. Nurs Clin North Am 2004; 39: 529-43.

15. Gruetzner H. (eds). A caregiver's guide and sourcebook. 3rd edit. John Wiley and Sons, 2001.

16. Morris J. The Clinical Dementia Rating (CDR): current version and scoring rules. Neurology 1993; 43: 2412-4.

17. Lim WS, Chong MS, Sahadevan S. Utility of the clinical dementia rating in Asian populations. Clin Med Res 2007; 5: 61-70.

18. Cohen-Mansfield J. Agitated behaviors in the elderly. II. Preliminary results in the cognitive deteriorated. J Am Geriatr Soc 1986; 34: 722-7.

19. Norberg A, Asplund K. Caregivers' experience of caring for severely demented patients in the terminal phase of life. West $\mathrm{J}$ Nurs Res 1990; 12: 75-84.

20. Hallberg IR, Norberg A. Staff's interpretation of the experiences behind vocally disruptive behaviour in severely demented patients and their feelings about it. An explorative study. Int $\mathrm{J}$ Aging Hum Dev 1990; 31: 297-307.

21. Razavi D, Delvaux N, Marchal S, Bredart A, Farvacques C, Paesmans M. The effects of a $24 \mathrm{~h}$ psycological training program on attitudes, communication skills and occupational stress in oncology: a randomised study. Eur J Cancer 1993; 29A: 1858-63.

22. Tsai SL, Crockett MS. Effect of relaxation training, combining imagery and meditation on the stress level of Chinese nurses working in modern hospitals in Taiwan. Issues Ment Health Nurs 1993; 14: 51-66.

23. Mimura C, Griffiths P. The effectiveness of current approaches to workplace stress management in the nursing profession: an evidence based literature review. Occup Environ Med 2003; 60: 10-5.

24. Berg A, Hansson UW, Hallberg IR. Nurses' creativity, tedium and burnout during one year of clinical supervision and implementa- 
tion of individually planned nursing care. J Adv Nurs 1994; 20 : 742-9.

25. Olsson A, Hallberg IR. Caring for demented people in their homes or in sheltered accommodation as reflected on by homecare staff during clinical supervision sessions. J Adv Nurs 1998; 27: 241-52.

26. Chang EM, Daly S, Hanckock KM et al. The relationships among workplace stressors, coping methods, demographic characteristics, and health in Australian nurses. J Prof Nurs 2006; 22 : 30-8.

27. Healy CM, McKay MF. Nursing stress: the effects of coping strategies and job satisfaction in a sample of Australian nurses. $J$ Adv Nurs 2000; 31: 681-8.

28. Charles SC, Warnecke RB, Wilbert JR, Lichtenberg R, DeJesus C. Sued and non-sued physicians: satisfaction, dissatisfaction, and sources of stress. Psychosomatic 1987; 28: 462-8.
29. Richardsen MA, Burke JR. Occupational stress and job satisfaction among Canadian physicians. Work Stress 1991; 5 : 301-13.

30. Lapane KL, Hughes CM. Considering the employee point of view: perceptions of job satisfaction and stress among nursing staff in nursing homes. J Am Med Dir Assoc 2007; 8: 8-13.

31. Lim J, Bogossian F, Ahern K. Stress and coping in Australian nurses: a systematic review. Int Nurs Rev 2010; 57: 22-31.

32. Grant LA, Kane RA, Potthoff SJ, Ryden M. Staff training and turnover in Alzheimer special care units: comparisons with nonspecial care units. Geriatr Nurs 1996; 17: 278-82.

33. Riggs J, Rantz M. A model of staff support to improve retention in long-term care. Nurs Admin 2001; 25: 43-54.

34. Phillips RM, Bonsteel SH. The faculty and information specialist partnership: stimulating student interest and experiential learning. Nurse Educ 2010; 35: 136-8. 